\title{
On the validity of the local diffusive paradigm in turbulent plasma transport
}

\author{
G. Dif-Pradalier* and P. H. Diamond \\ Center for Astrophysics and Space Sciences, UCSD, La Jolla, California 92093, USA
}

\author{
V. Grandgirard, Y. Sarazin, J. Abiteboul, X. Garbet, Ph. Ghendrih, and A. Strugarek \\ CEA, IRFM, F-13108 Saint-Paul-lez-Durance, France \\ S. Ku and C. S. Chang \\ Courant Institute of Mathematical Sciences, New York University, New York, New York 10012, USA
}

(Received 31 October 2009; published 3 August 2010)

\begin{abstract}
A systematic, constructive and self-consistent procedure to quantify nonlocal, nondiffusive action at a distance in plasma turbulence is exposed and applied to turbulent heat fluxes computed from the state-of-the-art full- $f$, flux-driven gyrokinetic GYSELA and XGC1 codes. A striking commonality is found: heat transport below a dynamically selected mesoscale has the structure of a Lévy distribution, is strongly nonlocal, nondiffusive, scale-free, and avalanche mediated; at larger scales, we report the observation of a self-organized flow structure which we call the " $\mathbf{E} \times \mathbf{B}$ staircase" after its planetary analog.
\end{abstract}

DOI: 10.1103/PhysRevE.82.025401

PACS number(s): 52.35.Py, 52.30.Cv, 52.35.Mw

When modeling transport processes in tokamak plasmas, the following oft-invoked-and questionable-assumption greatly prevails: widely separated regions of the plasma do not significantly interact with each other; and when they do, they do so diffusively. Recast differently, this paradigm states that any particle or heat flux can accurately be described using a set of local transport coefficientsdiffusivities or conductivities-which should be locally related through a generalized Fick's law to the thermodynamic forces which induce them.

Models with such assumptions will be referred to as local or quasilocal; they assume successive transport events to be either mutually independent or with both a short correlation and short memory; in other words transport events are random and accurately described by a classical Gauss-Markov process. In this line of thought, (i) cumulative, synergetic small events piling up and generating large scale, avalanchelike, temporally intermittent transport are disregarded on the widely believed basis that turbulence regulating $\mathbf{E} \times \mathbf{B}$ sheared flows would efficiently hinder large-scale, organized transport events, thus strongly favoring a local, diffusive transport paradigm. Nor is it taken into account the fact that (ii) a large localized event may have a significant aftereffect in the same region it occurred in.

Disappointment with local models is surprisingly mild, given the vast amount of commonplace observations showing inconsistencies between them and either perturbative (hot or cold pulse) experiments [1-5], Bohm-like scaling of the energy confinement time [6] or off-axis heating experiments $[7,8]$. Those inconsistencies with local transport models are usually dealt with by proposing phenomenological transport models which may include an ad hoc local critical threshold that switches between a slow and a fast transport channel; each of these channels remaining diffusive (local) in essence. The crude limitations of such models are, e.g., discussed in Ref. [9].

\footnotetext{
*gdifpradalier@ucsd.edu
}

Theoreticians have long alerted to the dubiety of local, diffusive approaches to nonlinear transport, using, e.g., continuous time random walks (CTRWs) [10] in as different areas as chaotic dynamics [11,12], geophysics, economics, financial mathematics [13], hydrodynamics [14], or even hydrology [15]. Early works in fusion research have also worked beyond local, diffusive models: either invoking (i) toroidal mode coupling to explain fast pulse propagation [16], (ii) self-organized criticality (SOC) [17-19] and the concept of marginal stability to connect nonlocal transport events to scale-free avalanches $[20,21]$ and explain ion profile stiffness, (iii) turbulence spreading where rapid pulse propagation is considered as a more general consequence of the nonlinear dynamics [22], or (iv) based on the fractional kinetic equation $[9,23]$, following pioneering works by Mandelbrot or Zaslavsky [24].

The present Rapid Communication builds upon these works, yet adopting a different standpoint. The attractiveness of the present approach rests with its extreme simplicity and its ability to provide a systematic, constructive and selfconsistent procedure to quantify nonlocal and nondiffusive behavior in complex geometry and realistic plasma parameters. Self-consistency is key to our approach: our analysis is based on a large database from the state-of-the-art full- $f$ fluxdriven gyrokinetic GYSELA [25] and XGC1 [26] codes: velocity fields, flows, and heat fluxes are fully self-consistent, differing from either CTRW or particle-following methods. Mathematically, this problem translates as follows. We wish to move from a local or quasilocal formalism: $Q(r)=-n(r) \chi(r) \nabla T(r), Q$ being the turbulent heat flux, $n$ the density, $\chi$ the turbulent diffusivity, and $T$ the temperatureeach of these three latter quantities are expressed locally at radius $r$-to a generalized heat transfer integral [27]:

$$
Q(r)=-\int \mathcal{K}_{r}\left(r, r^{\prime}\right) \nabla T\left(r^{\prime}\right) d r^{\prime},
$$

where the kernel $\mathcal{K}_{r}$ (including a mildly varying density dependence)_physically, a generalized diffusivity — is the 
crucial quantity. At this point, insofar as $\mathcal{K}_{r}$ is not specified, let us emphasize that no assumption of dominance of nonlocality over locality is made: Eq. (1) embeds both nonlocal and local $\left(\mathcal{K}_{r}\right.$ can be a Dirac distribution) formalisms. One goal of this paper is to provide a straightforward and systematic way to infer the form of $\mathcal{K}_{r}$, no assumptions on the nature of the dynamics (local or not) being made a priori. The basic idea is to interpret Eq. (1) as a convolution product and hence recast, using the convolution theorem, the heat transfer integral as a mere product in Fourier space: $\mathcal{F}\left(\mathcal{K}_{r}\right)=-\mathcal{F}(Q) / \mathcal{F}(\nabla T), \mathcal{F}$ being the radial Fourier transform. Thus, given any set of data, insofar as the turbulent heat flux $Q$ and the external thermodynamic forces are known, one can straightforwardly compute the (Fourier transform of) kernel $\mathcal{F}\left(\mathcal{K}_{r}\right)$.

To illustrate this idea, we performed this procedure on a vast sample of simulation data, encompassing significantly different plasma parameters. For an up-front comparison with local transport models, the quantities below are both flux-surface averaged and radially averaged over the central half of the simulation domain, as is the case for the normalized temperature gradient $R / L_{T}-$ known to quantify the strength of the turbulence drive: $Q \propto\left(R / L_{T}-5.9\right)^{1 / 2}$ [27]. We scan (i) the importance of SOC features while scanning this key control parameter from weak and close to marginal $\left(R / L_{T}=6.2\right)$ to moderate/strong $\left(R / L_{T}=7.5\right)$ turbulence regimes, (ii) the collisionality $\nu_{\star}$ over one order of magnitude in the tokamak-relevant low-collisional so-called "banana regime," from 0.05 up to 0.5 . At last, the system size, parametrized (iii) by the dimensionless $\rho_{\star}=\rho_{i} / a$ number spans from today's smallest tokamaks $\left(\rho_{\star}=1 / 128\right)$ down to tomorrow's largest ones (with the $\rho_{\star}=1 / 512$ Iter-like value). Here, $a$ denotes the minor radius and $\rho_{i}$ is the ion gyroradius; other typical plasma parameters read at mid radius $\rho=r / a=0.5$ : $T_{i}=T_{e}, \epsilon=r / R=0.17, q=1.4$, and $s=(r / q) d q / d r=0.78$. The safety factor $q$ is parabolic, $R=R_{0}+r \cos \theta$ is the major radius, $\epsilon$ the inverse aspect ratio, $T_{i}$ and $T_{e}$ the ion and electron temperatures and $\nu_{\star}=\epsilon^{-3 / 2} q R_{0} \nu_{i i} / v_{T}, v_{T}=(T / m)^{1 / 2}$ being the thermal velocity, $\nu_{i i}$ the ion-ion collision frequency. Also $\gamma_{E}=r \partial_{r}\left(E_{r} / r B\right)$ is the $\mathbf{E} \times \mathbf{B}$ shear rate. Typically, the $\rho_{\star}=1 / 256$ simulation with GYSELA involves over $6010^{9}$ grid points on a half-torus high-resolution $\left(r, \theta, \varphi, v_{\|}, \mu\right)=(512,512,128,128,16)$ mesh and the $\rho_{\star}=1 / 192$ simulation with XGC1 involves over $1310^{9}$ markers. The results are summarized in Fig. 1.

As the first remarkable feature from Fig. 1, (i) a universal pattern seems to arise, despite the widely different underlying plasma conditions. A generic exponential form $\mathcal{L}_{\gamma} \propto \exp \left(-\Delta\left|k_{r}\right|^{\gamma}\right)$ can indeed be tailored in each case to best fit the data, $k_{r}$ denoting the radial wave vector, normalized to $\rho_{i}$ at mid radius. The Fourier transforms are performed between $0.35 \leq \rho \leq 0.65$ after temporal average over a collision time. At this point, $\gamma$ and $\Delta$ are free parameters: $\gamma$ controlling the shape of the fit, $\Delta$ its width. A set of optimal $(\gamma, \Delta)$ pairs [e.g., in the case $\rho_{\star}=1 / 512$, pairs between $(\gamma, \Delta)=(0.9,18)$ and $(1.1,24)]$ may be found to equally well fit the data. Interestingly, in any case, $0.8<\gamma<1.2$, readily implying that (ii) the kernel $\mathcal{K}_{r}$ is a Lévy distribution with index $\gamma$. This result is especially attractive since Lévy distributions are characterized by a divergent second moment (infinite

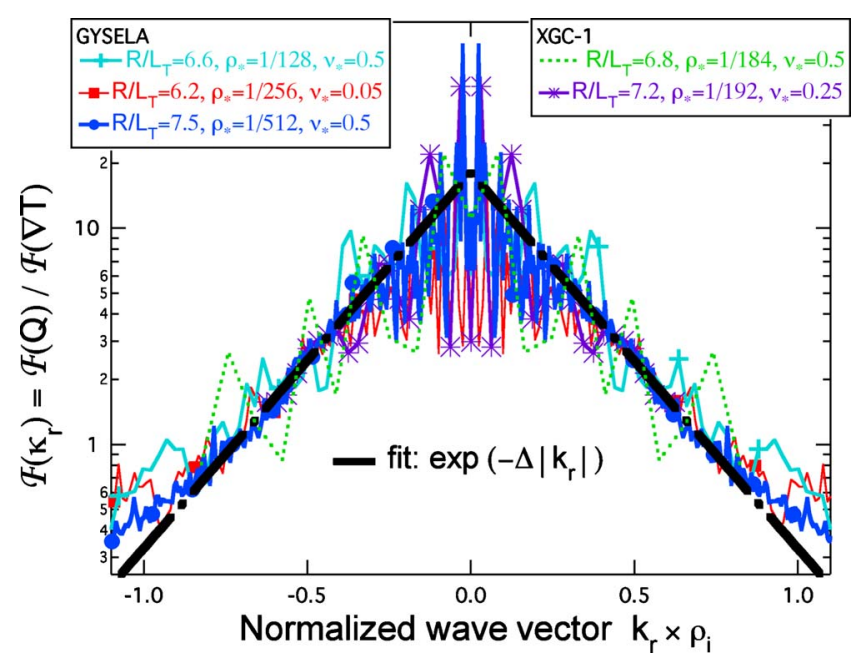

FIG. 1. (Color online) A Cauchy-Lorentz distribution robustly fits the kernel $\mathcal{F}\left(\mathcal{K}_{r}\right)$ throughout a wide span of parameters.

variance), making them choice candidates for modeling nonlocality.

At this point, let us strongly emphasize on the fact that finding the kernel $\mathcal{K}_{r}$ to be of Lévy type appeared selfconsistently, as an outcome of our procedure and that no preconceived hypothesis has led us to this conclusion. The current study, based on the self-consistent fluxes from GYSELA or XGC1, is strongly different in essence to procedures such as, e.g., those in Refs. [9,23], based on test particle following in a prechosen Lévy-like nondiffusive formalism. For the sake of simplicity, we now (iii) choose to discriminate among all optimal $(\gamma, \Delta)$ pairs by setting $\gamma=1$. This choice has several advantages: with limited loss of generality, it allows for physical intuition while remaining fully analytic. As a special case of Lévy distributions, the $\gamma=1$ case is trivially Fourier invertible, its inverse being the wellknown Cauchy-Lorentz distribution. Thus, $\mathcal{K}_{r}$ in real space now has the attractively simple analytic expression $(\Lambda$ being constant-see below):

$$
\mathcal{K}_{r}\left(r, r^{\prime}\right)=\frac{\Lambda}{\pi} \frac{\Delta / 2}{(\Delta / 2)^{2}+\left|r-r^{\prime}\right|^{2}} .
$$

As the Lorentzian width, parameter $\Delta$ may now further be interpreted as a radial influence length: a transport event happening at location $r$ can drive a flux up to a distance $\Delta$ from this event. One can now readily see that $\Delta$ does indeed take over a special relevance to assess the question of locality vs nonlocality: the larger $\Delta$, the stronger the nonlocality. As we now emphasize, $\Delta$ is found to be too large to allow reconciliation with local approaches: $\Delta \gg \ell_{c}$, the turbulence autocorrelation length.

For each simulation, the $\Delta$ values are straightforwardly evaluated as described above, by fitting $\mathcal{F}\left(\mathcal{K}_{r}\right)$ to $\mathcal{L}_{1}$. This analysis in Fourier space provides both the form of the kernel and a first estimate of the kernel width $\Delta$. To further buttress our argument, cross-check of these latter values has been performed in real space while minimizing the quantity: $\left|Q_{\text {simu }}^{2}-\left[\int \mathcal{K}_{r}\left(r, r^{\prime}\right) \nabla T\left(r^{\prime}\right) d r^{\prime}\right]^{2}\right|^{1 / 2}$, where $Q_{\text {simu }}$ denotes the 


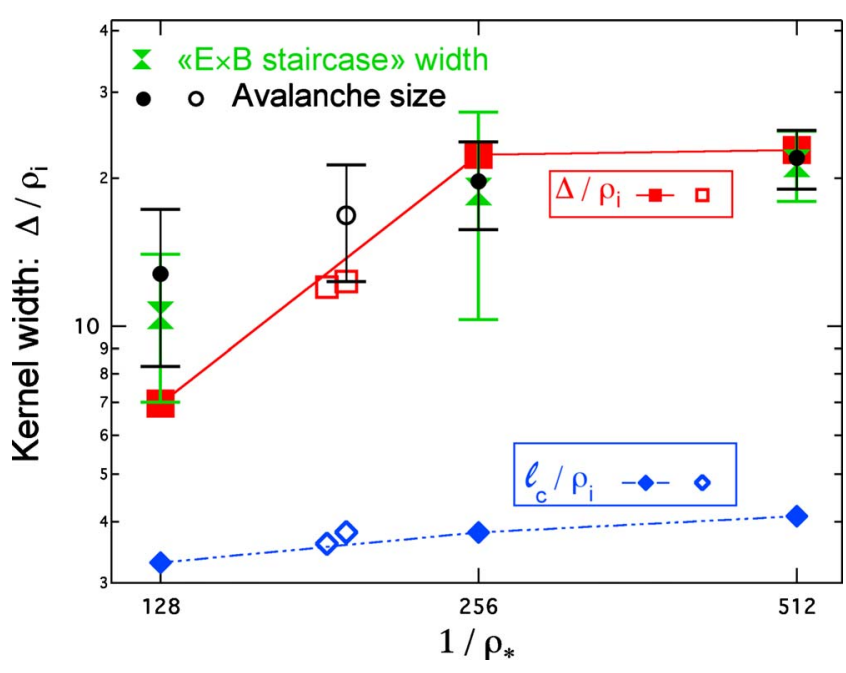

FIG. 2. (Color online) The "influence length" $\Delta$ is compared to the turbulence autocorrelation length $\ell_{c}$, the avalanche size and the "E $\times \mathbf{B}$ staircase" width between the jetlike structures in Fig. 3 (solid symbols, GYSELA; open symbols, XGC1).

simulated turbulent heat flux and $\mathcal{K}_{r}$ is given by Eq. (2). Through this minimization process, an optimal pair $(\Lambda, \Delta)$ is found for each simulation. When volume-averaging the turbulent heat flux, the issue of the nonlocality of kernel $\mathcal{K}_{r}$ is washed away and $\Lambda$-the strength parameter-scales as expected from the weak turbulence scaling: (i) $\chi^{\text {turb }} \propto \delta \phi^{2}$, the turbulence intensity, and (ii) transport near marginality is expressed [27], in the spirit of phase transitions, in terms of a critical threshold and critical exponent: $\chi^{\text {turb }} \propto\left(R / L_{T}-R / L_{T, c}\right)^{\alpha}$, with $R / L_{T, c}=5.9$ and $\alpha=1 / 2$. As a result, $\Lambda \propto\left\langle\delta \phi^{2}\right\rangle_{r}\left(\left\langle R / L_{T}\right\rangle_{r}-R / L_{T, c}\right)^{\alpha}$.

Regarding $\Delta$-the nonlocality parameter-its values, reported in Fig. 2, are (i) in close agreement (within 10\%) with the direct estimates from Fourier space and are (ii) mesoscale: well below the system size $a$, yet well above the turbulence autocorrelation length: $\ell_{c} \ll \Delta \ll a$. This length $\ell_{c}$ takes on a special relevance in local-like models; a similarly defined "influence length" would indeed range in these models from the ion Larmor radius $\rho_{i}$ up to $\ell_{c}$. Throughout the whole range of plasma parameters, as shown in Fig. 2, the influence lengths $\Delta$ estimated from Eqs. (1) and (2) are well beyond the correlation length of the fluctuations $\ell_{c}$, i.e., what one would expect from local models. If we further recall that $\mathcal{K}_{r}$ may equivalently be described by a second moment divergent Lévy distribution, reconciliation of this data with local or quasilocal models can only appear as fortuitous.

On the grounds of this difficult reconciliation, any transport model should endeavor to encompass the possibility for a nonlocal, nondiffusive action at a distance. A prominent manifestation of action at a distance in plasma turbulence are the commonly observed, intermittent, large-scale heat avalanches; in that spirit, the typical avalanche sizecomparable to the tail in the autocorrelation function $\mathcal{C}_{r}$-is compared in Fig. 2 to the kernel width $\Delta$ for each simulation. Close agreement is found, which interestingly grounds this intuitive nonlocal influence length $\Delta$-self-consistently obtained from actual heat fluxes- to the oft-invoked physics of self-organized near-critical transport, featuring long tails in

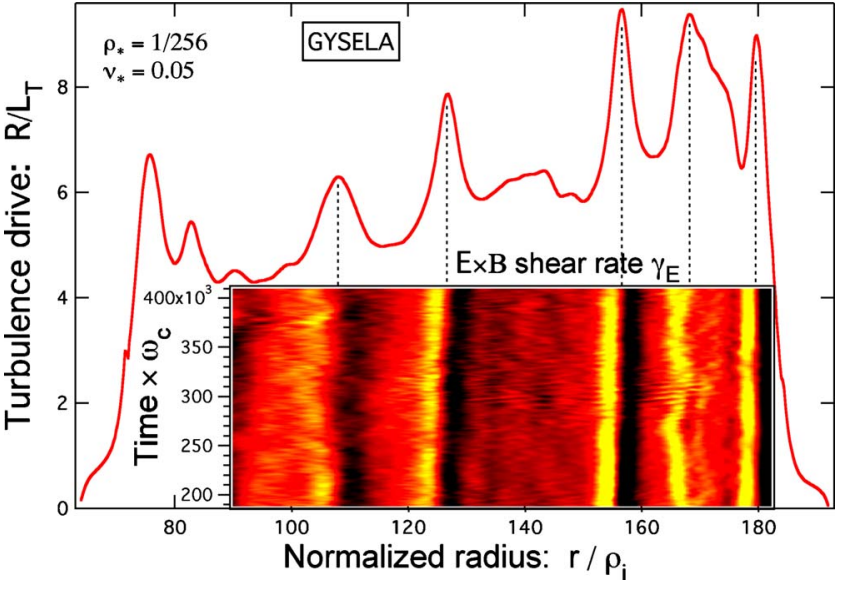

FIG. 3. (Color online) Corrugations of the mean temperature profile (averaged over $\nu_{i i}^{-1}$ ) correlate well with dynamically driven steady-standing $\mathbf{E} \times \mathbf{B}$ sheared flows which self-organize nonlinearly in a jetlike pattern: the " $\mathbf{E} \times \mathbf{B}$ staircase."

$\mathcal{C}_{r}, 1 / f$-type spectra [25,26], subcritical transport [27,25] and Hurst exponents of order unity [27].

Avalanches are often thought of as scale invariant; having this intrinsic "nonlocal length" saturating at mesoscale $\Delta$ $\ll a$ for large enough systems $\left(1 / \rho_{\star} \geq 256\right)$ is a remarkable manifestation of self-organization. Elucidating the mechanisms whereby this scale emerges is beyond the scope of this Letter. However, as a salient footprint of this influence length on the flow structure, strongly organized and persistent $\mathbf{E} \times \mathbf{B}$ sheared flows emerge and organize the turbulence into radial domains (Fig. 3, inset). The typical size of these domains (the spacing between the "jets") is shown as the $\mathbf{E} \times \mathbf{B}$ staircase width in Fig. 2; it also is in remarkable agreement with the avalanche size and the kernel influence length $\Delta$, reflecting the surprising tendency of the stochastic avalanche ensemble to self-organize in a jetlike pattern: few avalanches do indeed exist on scales larger than $\Delta$. Hence, any proposed mechanism will have to account for arresting the upscale of nonlocality to the system size and the emergence at this mesoscale $\Delta$ of a jetlike pattern of coherent structures of alternating sign: the " $\mathbf{E} \times \mathbf{B}$ staircase," see Fig. 3 , which we name after its planetary analog [28].

Note that the location of these jets does not seem to be tied to rational values of the safety factor $q$, as commonly emphasized. As a new perspective, one may ask whether, e.g., in such monotonic $q$-profile plasmas, the location of these jets could favor a transport barrier to nucleate.

A bigger picture starts to emerge, summarized in Table I, in which self-organization is present at all scales yet manifests itself differently at different scales; its most prominent feature being arguably the dynamical emergence of the mesoscale $\Delta$ : at scales smaller than $\Delta$, transport is scaleinvariant, avalanche-mediated, nonlocal and nondiffusive; at scales larger than $\Delta$, few genuinely scale-free avalanches exist; rather, strongly coherent and persistent flows organize the turbulence into a jetlike pattern, the $\mathbf{E} \times \mathbf{B}$ staircase. The system may thus either be seen as scale-invariant, nonlocal, nondiffusive (fractal or self-similar) on some scale or, equivalently, strongly organized on some other. Further dis- 
TABLE I. Turbulence-induced mesoscale $\Delta$ delineates the largescale organized flow pattern from small-scale avalanching.

\begin{tabular}{|c|c|}
\hline $\begin{array}{l}\text { ion Larmor gyroradius } \rho_{i} \\
\text { turbulence autocorrelation length } \ell_{c}\end{array}$ & $\begin{array}{l}\text { scale-free, non-local, } \\
\text { avalanche-mediated } \\
\text { dynamics }\end{array}$ \\
\hline $\begin{array}{l}\text { kernel width } \Delta \equiv \text { 'influence length' } \\
\sim \text { avalanche length } \\
\sim \text { 'E } \times \mathbf{B} \text { staircase' width }\end{array}$ & $\begin{array}{l}\text { self-selected } \\
\text { meso-scale dynamics }\end{array}$ \\
\hline $\begin{array}{c}\hat{\wedge} \text { (large systems) } \\
\text { temperature gradient length } L_{T} \\
\hat{\wedge} \\
\text { system size } \hat{a} \text { (simulation box })\end{array}$ & $\begin{array}{l}\text { persistent, jet-like } \\
\text { organised } \mathbf{E} \times \mathbf{B} \\
\text { structures }\end{array}$ \\
\hline
\end{tabular}

cussion of the $\mathbf{E} \times \mathbf{B}$ staircase, especially through discussion of the physical mechanism which sets the jet spacing-for its connection to mean flow shear, see Ref. [27]—is beyond the scope of the present paper and will be reported elsewhere.

So far, the heat flux $Q(r, t)$ has been temporally averaged over a collision time; we thus only addressed the spatial question of the nonlocal, nondiffusive (non-Gauss-Markov) behavior of $Q$. An equally interesting question is to now address its Markov (temporal) counterpart; essentially, two modeling choices are here possible:

$$
\begin{gathered}
Q(r, t)=-\int d r^{\prime} \mathcal{K}_{r}\left(r, r^{\prime}\right) \int d t^{\prime} \mathcal{K}_{t}\left(r^{\prime}, t, t^{\prime}\right) \nabla T\left(r^{\prime}, t^{\prime}\right), \\
Q(r, t)=-\iint d r^{\prime} d t^{\prime} \mathcal{K}_{r, t}\left(r, r^{\prime}, t, t^{\prime}\right) \nabla T\left(r^{\prime}, t^{\prime}\right) .
\end{gathered}
$$

Equation (3) is a straightforward follow-up from Eq. (1): $\mathcal{K}_{r}$ may still be given by Eq. (2) while $\mathcal{K}_{t}$ is now the unknown. Inversely, Eq. (4) is its generalization, the convolution theorem being applied to the 2D (radius, time) Fourier transform of $\mathcal{K}_{r, t}$. Both approaches have been investigated, giving similar conclusions. First, focusing on the formulation in Eq. (3), $\mathcal{K}_{t}$ is simply evaluated while either looking at (i) a definite radial position or (ii) averaging over a radial domain. Whatever the chosen position or the radial domain, no universal feature as in Fig. 1 could univocally be extracted from the available data. A similar conclusion holds when focusing on the formulation in Eq. (4), either looking at selected $k_{r}$ values or averaging over a selected domain in wave vector space.

In summary, (i) a simple, systematic, constructive and self-consistent procedure is proposed to quantify nonlocal, nondiffusive behavior based on the use of the convolution theorem. Fluxes are expressed as generalized heat transfer integrals for which (ii) an operational form is derived, through the finding of a universal Lévy-type kernel $\mathcal{K}_{r}$ - with no preconceived hypothesis regarding the nature of the heat transport. A strong (iii) nonlocal, nondiffusive spatial behavior is found, stemming from the self-organized, avalanchemediated transport. As a prominent feature of this selforganization, (iv) the dynamics is crucially determined through the emergence of a mesoscale $-\Delta-$ - which can altogether be interpreted as the kernel influence length, the avalanche size and the $\mathbf{E} \times \mathbf{B}$ staircase width. Scale-free avalanche-dominated nonlocal dynamics is found at sub- $\Delta$ scales; inversely, strongly organized, persistent jet-like patterns of $\mathbf{E} \times \mathbf{B}$ flows dominate above this mesoscale. At last, (v), the available data does not allow to conclude on the possibility of non-Markovian (memory) effects.

The authors want to thank Ch. Passeron, G. Latu, acknowledge collaboration with S. Klasky, and fruitful interactions at the Festival de Théorie, Aix-en-Provence, 2007 and 2009. This work benefited from the support of U.S. SciDAC Gps-TTBP, Grant No. DE-FC02-08ER54959; it was performed using HPC resources from NCSS Jaguar and Genci[CCRT/CINES/IDRIS], Grant No. 2009-052224.
[1] K. W. Gentle et al., Phys. Plasmas 2, 2292 (1995).

[2] P. Galli et al., Nucl. Fusion 39, 1355 (1999).

[3] P. Mantica et al., Phys. Rev. Lett. 82, 5048 (1999).

[4] F. Ryter et al., Nucl. Fusion 40, 1917 (2000).

[5] B. P. van Milligen et al., Nucl. Fusion 42, 787 (2002).

[6] C. C. Petty et al., Phys. Plasmas 2, 2342 (1995).

[7] T. C. Luce et al., Phys. Rev. Lett. 68, 52 (1992).

[8] C. C. Petty et al., Nucl. Fusion 34, 121 (1994).

[9] R. Sánchez et al., Phys. Plasmas 12, 056105 (2005).

[10] E. W. Montroll et al., J. Math. Phys. 6, 167 (1965).

[11] M. F. Shlesinger et al., Phys. Rev. Lett. 58, 1100 (1987).

[12] J. Klafter et al., Phys. Rev. A 35, 3081 (1987).

[13] B. B. Mandelbrot, The Fractal Geometry of Nature (Freeman, New York, 1983).

[14] T. Hwa and M. Kardar, Phys. Rev. A 45, 7002 (1992).
[15] B. B. Mandelbrot et al., Water Resour. Res. 4, 909 (1968).

[16] X. Garbet et al., Nucl. Fusion 34, 963 (1994).

[17] P. H. Diamond et al., Phys. Plasmas 2, 3640 (1995).

[18] B. A. Carreras et al., Phys. Plasmas 3, 2903 (1996).

[19] X. Garbet et al., Phys. Plasmas 5, 2836 (1998).

[20] P. A. Politzer, Phys. Rev. Lett. 84, 1192 (2000).

[21] P. Beyer et al., Phys. Rev. Lett. 85, 4892 (2000).

[22] T. S. Hahm et al., Phys. Plasmas 12, 090903 (2005).

[23] D. del-Castillo-Negrete, Phys. Plasmas 13, 082308 (2006).

[24] G. M. Zaslavsky, Hamiltonian Chaos and Fractional Dynamics (Oxford University Press, Oxford, 2005).

[25] Y. Sarazin et al., Nucl. Fusion 50, 054004 (2010).

[26] S. Ku et al., Nucl. Fusion 49, 115021 (2009).

[27] G. Dif-Pradalier et al., Phys. Rev. Lett. 103, 065002 (2009).

[28] M. E. McIntyre et al., Nature (London) 305, 593 (1983). 